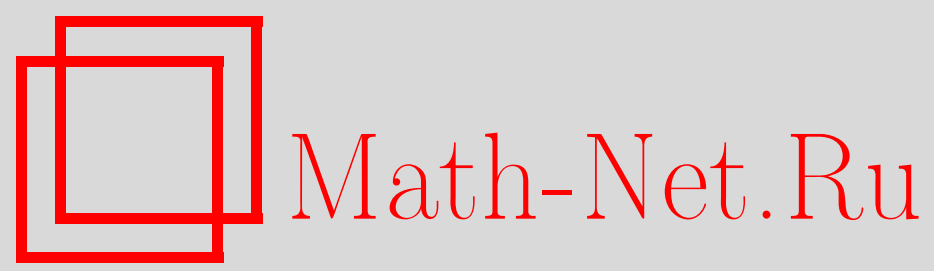

Р. Р. Исмагилова, Решение полного матричного аналога обобщённого уравнения Абеля с постоянными коэффициентами, Вестн. Сам. гос. техн. ун-та. Сер. Физ.-мат. науки, 2011, выпуск 1(), 93-98

DOI: https://doi.org/10.14498/vsgtu959

Использование Общероссийского математического портала Math-Net.Ru подразумевает, что вы прочитали и согласны с пользовательским соглашением http://www.mathnet.ru/rus/agreement

Параметры загрузки:

IP: 54.89 .56 .158

26 апреля 2023 г., $17: 01: 52$ 
УДК 517.968.78

\section{РЕШЕНИЕ ПОЛНОГО МАТРИЧНОГО АНАЛОГА ОБОБЩЁННОГО УРАВНЕНИЯ АБЕЛЯ С ПОСТОЯННЫМИ КОЭФФИЦИЕНТАМИ}

\section{Р. Р. Исмагилова}

Самарский государственный технический университет, 443100, Самара, ул. Молодогвардейская, 244.

E-mail: isriri@mail.ru

Рассмотрена система обобщённых интегральных уравнений Абеля с постоянными коэфбициентами в матричной форме в терминах интегральных операторов Римана-Лиувилля матричного порядка на отрезке. Обоснована её редукиия $к$ системе сингулярных интегральных уравнений. Решение этой системъ найдено в явном виде в случае коммутативных матрии, простой структуры.

Ключевые слова: дробное исчисление, функиии матричного аргумента, интегродифференциальные операторы матричного порядка, система обобщённых интегральных уравнений Абеля.

Пусть $\lambda \in C, f(x)$ - измеримая, в общем случае, комплексно-значная функция с областью определения $D(f) \subset \Omega=[a, b]$, где $-\infty<a<b<+\infty$. Пусть $c$ - произвольная точка отрезка $\Omega: a \leqslant c \leqslant b$.

Интегро-дифференциальный оператор Римана-Лиувилля порядка $\lambda$ определяется так [1]:

$$
I_{c x}^{\lambda} f \equiv D_{c x}^{-\lambda} f=\left[\begin{array}{ll}
\frac{\operatorname{sign}(x-c)}{\Gamma(\lambda)} \int_{c}^{x} \frac{f(t) d t}{|x-t|^{1-\lambda}}, & \operatorname{Re} \lambda>0 ; \\
\operatorname{sign}(x-c)\left(\frac{\partial}{\partial x}\right)^{n} I_{c x}^{n+\lambda} f, & \operatorname{Re} \lambda \leqslant 0, n=[-\operatorname{Re} \lambda]+1,
\end{array}\right.
$$

где $\Gamma(\lambda)$ - гамма-функция Эйлера, $[\cdot]$ - целая часть числа. При $a \leqslant c<x \leqslant b$ $(1)$ определяет левостронний интегро-дифференциальный оператор $I_{c+}^{\lambda}$, а при $a \leqslant x<c \leqslant b-$ правосторонний оператор $I_{c-}^{\lambda}[2]$. При $\lambda=0$ в силу определения (1) $I_{c x}^{0} \equiv D_{c x}^{0} \equiv I$ - тождественный оператор.

Обозначим $M_{n}$ - множество постоянных матриц порядка $n, \Lambda(G)$ - спектр матрицы $G \in M_{n}, \lambda_{i} \in \mathbb{C}$ - собственные значения матрицы $G, i=1,2, \ldots, n$. Обозначим $\mathbb{C}_{+}=\{z \in \mathbb{C}: \operatorname{Re} z>0\}$ и $\mathbb{C}_{-}=\{z \in \mathbb{C}: \operatorname{Re} z \leqslant 0\}$.

В соответствии с определением, введенным в работах $[3,4]$, для матрицы $G \in M_{n}$ действие матричного оператора дробного интегро-дифференцирования Римана-Лиувилля на функцию $f(x)=\left(f_{1}, f_{2}, \ldots, f_{n}\right)^{\top}$ даётся формулой

$$
\left(I_{c x}^{G}\right) f \equiv\left(D_{c x}^{-G}\right) f=\left.\sum_{k=1}^{s} \psi^{k}(G) \sum_{n=0}^{m_{k}-1} \frac{\left(G-\lambda_{k} E\right)^{n}}{n !}\left(\frac{d^{n}}{d \lambda^{n}} \frac{I_{c x}^{\lambda}}{\psi^{k}(\lambda)}\right) f\right|_{\lambda=\lambda_{k}},
$$

где $\psi^{k}(\lambda)=\left(\lambda-\lambda_{k}\right)^{-m_{k}} \prod_{k=1}^{s}\left(\lambda-\lambda_{k}\right)^{m_{k}}, \lambda_{k} \in \Lambda(G)$.

Рина Ринатовна Исмагилова, аспирант, каф. прикладной математики и информатики. 
Если $\Lambda(G) \subset \mathbb{C}_{+}$, то $I_{c x}^{G} f$ определяет матричный аналог интегрального оператора Римана-Лиувилля, в частности, при $c=a$ и $x>a$ имеем левосторонний интегральный оператор $I_{a+}^{G} f$ матричного порядка, а при $c=b$ и $x<b$-правосторонний оператор $I_{b-}^{G} f$. Если $\Lambda(G) \subset \mathbb{C}_{-}$, то формула $(2)$ определяет матричный аналог дифференциального оператора Римана-Лиувилля. А при $\Lambda(G) \subset C$ имеем матричный аналог интегро-дифференциального оператора Римана-Лиувилля.

Пусть матрицы $A, B, G \in M_{n}$ и пусть матрица $G$ является матрицей простой структуры, а $\Lambda(G) \subset \mathbb{R}_{+}$, где $\mathbb{R}_{+}=\{x: x \in \mathbb{R}, x>0\}$. Систему интегральных уравнений

$$
A I_{a+}^{G} \varphi+B I_{b-}^{G} \varphi=f(x),
$$

где $\varphi(x)$ - искомая, а $f(x)$ - заданная $n$-мерные вектор-функции, будем называть системой обобщённых интегральных уравнением Абеля на отрезке $[a, b]$.

Если одна из матриц $A$ или $B$ нулевая, получим матричные аналоги классических уравнений Абеля с правосторонним или левосторонним интегралом матричного порядка, рассмотренные в работах $[5,6]$.

Пусть $\operatorname{det} A \neq 0$. Тогда система интегральных уравнений (3) может быть записана в виде

$$
E I_{a+}^{G} \varphi+P I_{b-}^{G} \varphi=g(x),
$$

где $P=A^{-1} B, g(x)=A^{-1} f(x)$.

С помощью свойств интегро-дифференциальных операторов матричного порядка $[6,7]$, система уравнений Абеля (3) редуцируется к системе интегральных уравнений Фредгольма первого рода

$$
\Gamma^{-1}(G) \int_{a}^{b}(C+D \operatorname{sign}(x-t))|x-t|^{G-E} \varphi(t) d t=g(x),
$$

где $C=(E+P) / 2, D=(E-P) / 2$, а $\Gamma(G)$ - гамма-функция Эйлера матричного аргумента. Заметим, что когда $D$ - нулевая матрица, уравнение (5) является матричным аналогом уравнения Карлемана $[2,8]$.

Рассмотрим случай коммутативных матриц $G$ и $P$, считая их матрицами простой структуры. Известно [9], что в этом случае существует невырожденное преобразование с матрицей $T$, приводящее матрицы $G$ и $P$ одновременно к диагональным матрицам. Пусть $G=T^{-1} \Lambda_{G} T, P=T^{-1} \Lambda_{P} T$, где $\Lambda_{G}, \Lambda_{P}$ - соответствующие диагональные матрицы. Тогда $(x-t)^{G-E}=$ $=T^{-1}|x-t|^{-\Lambda_{G-E}} T$. Обозначим $h(x)=T g(x)$ и $\psi(x)=T \varphi(x)$, где $\psi(x)=$ $=\left(\psi_{1}(x), \psi_{2}(x), \ldots, \psi_{k}(x)\right)^{\top}, h(x)=\left(h_{1}(x), h_{2}(x), \ldots, h_{k}(x)\right)^{\top}$. Тогда система уравнений $(5)$ при $\Lambda(P)=\left\{p_{k}\right\}, k=1,2, \ldots, n$, расщепляется на $n$ уравнений вида

$$
\frac{1}{2} \Gamma^{-1}\left(\lambda_{k}\right) \int_{a}^{b}\left(\left(1+p_{k}\right)+\left(1-p_{k}\right) \operatorname{sign}(x-t)\right)|x-t|^{\lambda_{k}-1} \psi_{k}(t) d t=h_{k}(x) .
$$

Известно [8], что порядок особенностей решений уравнений системы (6) определяется числом

$$
\theta_{k}=\arg H_{k}(a)=2 \arg \left(1-p_{k} e^{\left(1-\lambda_{k}\right) \pi i}\right) \quad(k=1,2, \ldots, n), \quad 0<\theta<2 \pi,
$$


где

$$
H_{k}(a)=\frac{1-p_{k} e^{\left(1-\lambda_{k}\right) \pi i}}{1-p_{k} e^{-\left(1-\lambda_{k}\right) \pi i}}
$$

- коэффициент задачи Римана, соответствующей каждому уравнению системы (6).

Вернёмся к системе интегральных уравнений (4). Покажем, что в случае коммутативных матриц $G$ и $P$ она редуцируется к системе сингулярных интегральных уравнений.

ЛЕмма 1. Пусть $\Lambda(G) \subset(0,1)$,

$$
(S \varphi)(x)=\frac{1}{\pi} \int_{a}^{b} \frac{\varphi(t)}{t-x} d t
$$

- сингулярный интегральный оператор, $r_{b}=b-x$. Тогда матричные итегральные операторы $I_{a+}^{G}, I_{b-}^{G}$ и оператор $S$ связаны соотношением

$$
I_{b-}^{G} \varphi=\cos (G \pi) I_{a+}^{G} \varphi+\sin (G \pi) r_{b}^{G} S r_{b}^{-G} I_{a+}^{G} \varphi \quad\left(\varphi \in L_{p}, p \geqslant 1\right)
$$

Доказ а тельств о. Для простоты ограничимся множеством $M_{2}$. Пусть $G \in M_{2}$ и является матрицей простой структуры. Тогда, используя определение (2) и определение функции матричного аргумента [9], правую часть (8) можно записать в виде

$$
\begin{gathered}
\left(\frac{G-\lambda_{2} E}{\lambda_{1}-\lambda_{2}} \cos \lambda_{1} \pi+\frac{G-\lambda_{1} E}{\lambda_{2}-\lambda_{1}} \cos \lambda_{2} \pi\right)\left(\frac{G-\lambda_{2} E}{\lambda_{1}-\lambda_{2}} I_{a+}^{\lambda_{1}}+\frac{G-\lambda_{1} E}{\lambda_{2}-\lambda_{1}} I_{a+}^{\lambda_{2}}\right) \varphi+ \\
+\left(\frac{G-\lambda_{2} E}{\lambda_{1}-\lambda_{2}} \sin \lambda_{1} \pi+\frac{G-\lambda_{1} E}{\lambda_{2}-\lambda_{1}} \sin \lambda_{2} \pi\right)\left(\frac{G-\lambda_{2} E}{\lambda_{1}-\lambda_{2}} r_{b}^{\lambda_{1}}+\frac{G-\lambda_{1} E}{\lambda_{2}-\lambda_{1}} r_{b}^{\lambda_{2}}\right) \times \\
\quad \times S\left(\frac{G-\lambda_{2} E}{\lambda_{1}-\lambda_{2}} r_{b}^{-\lambda_{1}}+\frac{G-\lambda_{1} E}{\lambda_{2}-\lambda_{1}} r_{b}^{-\lambda_{2}}\right)\left(\frac{G-\lambda_{2} E}{\lambda_{1}-\lambda_{2}} I_{a+}^{\lambda_{1}}+\frac{G-\lambda_{1} E}{\lambda_{2}-\lambda_{1}} I_{a+}^{\lambda_{2}}\right) \varphi
\end{gathered}
$$

Используя свойства идемпотентов матрицы $G$, легко получить

$$
\begin{aligned}
& \frac{G-\lambda_{2} E}{\lambda_{1}-\lambda_{2}}\left(\cos \left(\lambda_{1} \pi\right) I_{a+}^{\lambda_{1}}+\sin \left(\lambda_{1} \pi\right) r_{b}^{\lambda_{1}} S r_{b}^{-\lambda_{1}} I_{a+}^{\lambda_{1}}\right) \varphi+ \\
& +\frac{G-\lambda_{1} E}{\lambda_{2}-\lambda_{1}}\left(\cos \left(\lambda_{2} \pi\right) I_{a+}^{\lambda_{2}}+\sin \left(\lambda_{2} \pi\right) r_{b}^{\lambda_{2}} S r_{b}^{-\lambda_{2}} I_{a+}^{\lambda_{2}}\right) \varphi=I_{b-}^{G} \varphi
\end{aligned}
$$

откуда следует формула (8).

Лемма 2. Система интегральных уравнений Абеля (4) в классе суммируемых функиий эквивалентна системе сингулярных интегральных уравнений

$$
A_{1} \Phi(x)+\frac{A_{2}}{\pi} \int_{a}^{b} \frac{\Phi(t) d t}{t-x}=r_{b}^{-G} g(x),
$$

где $A_{1}=E+P \cos (G \pi), A_{2}=P \sin (G \pi), \Phi(x)=r_{b}^{-G} I_{a+}^{G} \varphi$. 
Доказательство. В системе интегральных уравнений (4) заменим матричный интегральный оператор $I_{b-}^{G}$ его выражением по формуле (8). Тогда

$$
E I_{a+}^{G} \varphi+P \cos (G \pi) I_{a+}^{G} \varphi+P \sin (G \pi) r_{b}^{G} S r_{b}^{-G} I_{a+}^{G} \varphi=g(x) .
$$

Умножая левую и правую части этого уравнения на матрицу $r_{b}^{-G}$ слева и вводя вектор $\Phi(x)=r_{b}^{-G} I_{a+}^{G} \varphi$, получим систему интегральных уравнений (9):

$$
\left(A_{1}+A_{2} S\right) \Phi(x)=r_{b}^{-G} g(x) .
$$

Очевидно, что с помощью матрицы $T$ система интегральных уравнений (4) расщепляется на $n$ уравнений вида

$$
I_{a+}^{\lambda_{k}} \psi_{k}+p_{k} I_{b-}^{\lambda_{k}} \psi_{k}=h_{k}(x)
$$

а система уравнений (9) расщепляется на

$$
\left(1+p_{k} \cos \left(\lambda_{k} \pi\right)\right) \Psi_{k}+\pi^{-1} p_{k} \sin \left(\lambda_{k} \pi\right) \int_{a}^{b} \frac{\Psi_{k}(t)}{t-x} d t=r_{b}^{-\lambda_{k}} h_{k},
$$

где $\Psi_{k}(x)$ - компонента вектора $\Psi(x)=T \Phi(x)=\left(\Psi_{1}(x), \Psi_{2}(x), \ldots, \Psi_{n}(x)\right)^{\top}$.

Используя известные решения уравнений (11) [8], нетрудно найти выражения дробных интегралов от компонент вектора $\psi(x)$ :

$$
\begin{aligned}
I_{a+}^{\lambda_{k}} \psi_{k} & =r_{b}^{\lambda_{k}} \Psi_{k}=\frac{1-p_{k} \cos \left(1-\lambda_{k}\right) \pi}{l_{k}} h_{k}(x)+\frac{c_{k}}{r_{a}^{\theta_{k} /(2 \pi)} r_{b}^{1-\lambda_{k}-\theta_{k} /(2 \pi)}}- \\
& -\frac{p_{k} \sin \left(1-\lambda_{k}\right) \pi}{l_{k} \pi r_{a}^{\theta_{k} /(2 \pi)} r_{b}^{1-\lambda_{k}-\theta_{k} /(2 \pi)}} \int_{a}^{b} \frac{(b-t)^{1-\lambda_{k}-\theta_{k} /(2 \pi)}(t-a)^{\theta_{k} /(2 \pi)}}{t-x} h_{k}(t) d t
\end{aligned}
$$

в случае $\theta_{k}<2 \pi\left(1-\lambda_{k}\right)$ и

$$
\begin{aligned}
I_{a+}^{\lambda_{k}} & \psi_{k} \\
& =r_{b}^{\lambda_{k}} \Psi_{k}=\frac{\left(1-p_{k}\right) \cos \left(1-\lambda_{k}\right) \pi}{l_{k}} h_{k}(x)-\frac{p_{k} \sin \left(1-\lambda_{k}\right) \pi}{l_{k} \pi}- \\
& -r_{a}^{1-\theta_{k} /(2 \pi)} r_{b}^{-\left(1-\lambda_{k}-\theta_{k} /(2 \pi)\right)} \int_{a}^{b} \frac{\psi_{k}(t) d t}{(t-a)^{1-\theta_{k} /(2 \pi)}(b-t)^{\theta_{k} /(2 \pi)-1+\lambda_{k}}(t-x)}
\end{aligned}
$$

в случае $\theta_{k} \geqslant 2 \pi\left(1-\lambda_{k}\right)$, где $l_{k}=1-2 p_{k} \cos \left(1-\lambda_{k}\right) \pi+p_{k}^{2}, r_{a}=x-a, c_{k}$ произвольная постоянная.

Для определения компонент вектора $\psi(x)$ остаётся решить обычные уравнения Абеля. Таким образом, уравнение (10) будет иметь в классе гёльдеровских функций с интегрируемыми особенностями на концах единственное решение тогда и только тогда, когда $\arg \left(1-p_{k} e^{\left(1-\lambda_{k}\right) \pi i}\right) \geqslant\left(1-\lambda_{k}\right) \pi$. Следовательно, справедлива следующая лемма.

Лемма 3. Система уравнений (10) разрешима в классе вектор-функций $H^{*}$ при любых правых частях $h_{k}(x) \in H_{\lambda_{k}}^{*}$, если $p_{k}>0, k=1,2, \ldots, n$. Решение единственно и его компоненты определяются формулами

$$
\psi_{k}(x)=l_{k}^{-1} \Gamma^{-1}\left(\lambda_{k}\right) D_{a+}^{\lambda_{k}}\left(h_{k}-p_{k} Z_{k} D_{a+}^{1-\lambda_{k}} I_{b-}^{1-\lambda_{k}} Z_{k}^{-1} h_{k}\right),
$$


где

$$
Z_{k}(x)=r_{a}^{2-\lambda_{k}-\theta_{k} /(2 \pi)} r_{b}^{-\left(1-\lambda_{k}-\theta_{k} /(2 \pi)\right)}
$$

ЗАмечание. Определение классов $H^{*}$ и $H_{\lambda_{k}}^{*}$ см. в [2].

Окончательный результат сформулируем в виде теоремы.

Теорема. Пусть в системе обобщённых интегральных уравнений Абеля (4) матрицы $G$ и $P$ коммутативны и являются матрицами простой структуры такими, что $\Lambda(G) \subset(0,1), \Lambda(P) \subset \mathbb{R}_{+}$. Пусть вектор-функция $g(x)$ такова, что компоненты вектора $h(x)=T g(x)$ принадлежсат классам бункиии $H_{\lambda_{k}}^{*}, k=1,2, \ldots, n$, где $\lambda_{k} \in \Lambda(G)$ - собственные значения матри-

иљь $G$, а $T$-матрица преобразования $G \kappa$ диагональному виду $\Lambda_{G}=T G T^{-1}$. Тогда единственное решение системы уравнений (4) в классе вектор-фууниий $H^{*}$ имеет вид

$$
\psi(x)=L^{-1} \Gamma^{-1}(G) D_{a+}^{G}\left(E-P Z(x) D_{a+}^{E-G} I_{b-}^{E-G} Z^{-1}(x)\right) g(x),
$$

где матрица $Z(x)=T^{-1} \operatorname{diag}\left(Z_{1}(x), Z_{2}(x), \ldots, Z_{n}(x)\right) T$, a $Z_{k}(x)$ определенъь $8(12)$.

\section{БИБЛИОГРАФИЧЕСКИЙ СПИСОК}

1. Нахушев А.М. Дробное исчисление и его применение. М.: Физмалит, 2003. 272 с. [Nakhushev A. M. Fractional Calculus and Its Applications. Moscow: Fizmatlit, 2003. 272 pp.]

2. Самко С.Г., Килбас А.А., Маричев О.И. Интегралы и производные дробного порядка и некоторые их приложения. Минск: Наука и техника, 1987. 688 с с. [Samko S. G., Kilbas A.A., Marichev O.I. Integrals and derivatives of fractional order and some of their applications. Minsk: Nauka i Tekhnika, 1987. 688 pp.]

3. Андреев A. А. Нелокальные краевые задачи для одной модельной вырождающейся системы гиперболического типах/ В сб.: Краевые задачи для уравнений математической физики. Куйбышев: Куйбыш. гос. пед. ин-т, 1990. С. 3-7. [Andreev A. A. Non-local boundary value problems for one degenerate model system of hyperbolic type / In: Boundary value problems for equations of mathematical physics. Kuibyshev: Kuibysh. Gos. Ped. In-t, 1990. Pp. 3-7].

4. Андреев A.А. Об одном обобщении операторов дробного интегро-дифференцирования и его приложениях / В сб.: Интегральные уравнения и краевые задачи математической физики: Матер. Всессоюзной конф. Владивосток. Владивосток, 1990. С. 91. [Andreev A. A. On one generalization of fractional integro-differentiation operators and its applications / In: Integral Equations and Boundary Value Problems of Mathematical Physics. Vladivostok, 1990. Pp. 91].

5. Андреев A.A., Огородников E.Н. Матричные интегродифференциальные операторы и их применение // Вестн. Сам. гос. техн. ун-та. Сер. Физ.-мат. науки, 1999. №7. C. 27-37. [Andreev A. A., Ogorodnikov E. N. Matrix integro-differential operators and their application // Vestn. Samar. Gos. Tekhn. Univ. Ser. Fiz.-Mat. Nauki, 1999. no. 7. Pp. 27-37].

6. Исмагилова Р. Р. Свойства оператора обращения матричного уравнения Абеля // Вестн. Сам. гос. техн. ун-та. Сер. Физ.-мат. науки, 2010. Т. 5(21). C. 237-243. [Ismagiliva R. R. Properties of inversion operator of the Abel matrix equation// Vestn. Samar. Gos. Tekhn. Univ. Ser. Fiz.-Mat. Nauki, 2010. Vol.5(21). Pp. 237-243].

7. Исмагилова Р. Р. О некоторых свойствах операторов дробного интегро-дифференцирования матричного порядка / В сб.: Тр. Седъмой Всероссийской научн. конф. с междунар. участием Ч. 3: Дифференциальные уравнения и краевые задачи / Мат. моделирование и краевые задачи. Самара: СамГТУ, 2010. С. 129-132. [Ismagiliva R. R. On some 
properties of fractional integro-differentiation operators matrix order / In: Proceedings of the Seventh All-Russian Scientific Conference with international participation. Part 3 / Matem. Mod. Kraev. Zadachi. Samara: SamGTU, 2010. Pp. 129-132].

8. Гахов Ф.Д. Краевые задачи. М.: Физматлит, 1963. 640 с.; англ. пер.: Gakhov F.D. Boundary Value Problems. Oxford: Pergamon Press, 1966. 561 pp.

9. Гантмахер Ф.Р. Теория матриц. М.: Наука, 1988. 549 с. [Gantmakher F. R. Theory of matrices. Moscow: Nauka, 1988. 549 pp.]

Поступила в редакцию 20/XII/2010;

в окончательном варианте $-27 / \mathrm{II} / 2011$.

MSC: 47G20, 26A33

THE SOLUTION OF THE FULL MATRIX ANALOGUE OF THE GENERALIZED ABEL EQUATION WITH CONSTANT COEFFICIENTS

\section{R. R. Ismagilova}

Samara State Technical University,

244, Molodogvardeyskaya st., Samara, 443100, Russia.

E-mail: isriri@mail.ru

The system of generalized integral Abel equations in the matrix form with constant coefficients on the segment. Was considered at the terms of the integral RiemannLiouville operators of matrix order. It's reduction to the system of singular integral equations was founded. Solution of this system was found for the case of the commutative matrices of the simple structure in the explicit form.

Key words: fractional calculus, functions of matrix argument, integro-differential operators matrix order, system of generalized Abel integral equation.

Original article submitted 20/XII/2010; revision submitted $27 / \mathrm{II} / 2011$.

Rina R. Ismagilova, Postgraduate Student, Dept. of Applied Mathematics \& Computer Science. 